\title{
Fast and accurate analysis of large-scale composite structures with the parallel multilevel fast multipole algorithm
}

\author{
Özgür Ergül ${ }^{1, *}$ and Levent Gürel ${ }^{2,3}$ \\ ${ }^{1}$ Department of Mathematics and Statistics, University of Strathclyde, Glasgow G11XH, UK \\ ${ }^{2}$ Department of Electrical and Electronics Engineering, Bilkent University, Bilkent TR-06800, Ankara, Turkey \\ ${ }^{3}$ Computational Electromagnetics Research Center (BiLCEM), Bilkent University, Bilkent TR-06800, Ankara, Turkey \\ *Corresponding author: ozgur.ergul@strath.ac.uk
}

Received October 22, 2012; accepted January 29, 2013;

posted February 6, 2013 (Doc. ID 178408); published February 26, 2013

\begin{abstract}
Accurate electromagnetic modeling of complicated optical structures poses several challenges. Optical metamaterial and plasmonic structures are composed of multiple coexisting dielectric and/or conducting parts. Such composite structures may possess diverse values of conductivities and dielectric constants, including negative permittivity and permeability. Further challenges are the large sizes of the structures with respect to wavelength and the complexities of the geometries. In order to overcome these challenges and to achieve rigorous and efficient electromagnetic modeling of three-dimensional optical composite structures, we have developed a parallel implementation of the multilevel fast multipole algorithm (MLFMA). Precise formulation of composite structures is achieved with the so-called "electric and magnetic current combined-field integral equation." Surface integral equations are carefully discretized with piecewise linear basis functions, and the ensuing dense matrix equations are solved iteratively with parallel MLFMA. The hierarchical strategy is used for the efficient parallelization of MLFMA on distributed-memory architectures. In this paper, fast and accurate solutions of large-scale canonical and complicated real-life problems, such as optical metamaterials, discretized with tens of millions of unknowns are presented in order to demonstrate the capabilities of the proposed electromagnetic solver. @ 2013 Optical Society of America
\end{abstract}

OCIS codes: $\quad 290.0290,230.0230$.

\section{INTRODUCTION}

Recent developments in parallel implementations of fast algorithms, especially the multilevel fast multipole algorithm (MLFMA) [1-3], have enabled the solution of various real-life problems in electromagnetics [3-23]. Scattering and radiation problems involving large-scale three-dimensional structures can be formulated with surface integral equations and converted into dense matrix equations via discretization. These equations can be solved iteratively and the required matrixvector multiplications are performed efficiently and accurately via MLFMA. With an efficient parallelization of MLFMA, it becomes possible to solve complex problems discretized with hundreds of millions of unknowns on relatively inexpensive computers with distributed-memory architectures.

Realistic problems often involve composite structures with multiple dielectric and/or metallic parts. Using the equivalence theorem, such a composite problem involving a complicated structure can be decomposed into subproblems, where unknowns are equivalent surface currents defined at boundaries separating different regions. Various surface formulations can be derived by combining boundary conditions. The electric and magnetic current combined-field integral equation (JMCFIE) [24-30] is one of the suitable formulations for composite structures. This formulation can be discretized with the conventional Rao-Wilton-Glisson (RWG) functions
[31] on planar triangles, leading to well-conditioned matrix equations that can be solved iteratively via MLFMA.

Parallelization of MLFMA is not trivial due to the complicated structure of this algorithm. Recent efforts have mainly focused on increasing the parallelization efficiency and the

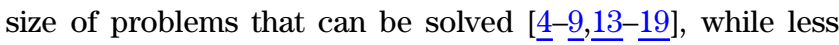
attention has been paid to material properties of objects [10-12,20-23]. The aim of this paper is to present a parallel implementation of MLFMA for composite structures with diverse material properties. We extend the hierarchical strategy, which was originally developed for perfect electric conductors (PECs) [ㄱ, 13] and recently applied to homogeneous dielectric objects [21-23], to composite structures with coexisting multiple dielectric and/or metallic parts. We demonstrate the capabilities of the extended implementation on large-scale canonical problems (such as layered spherical structures) and real-life objects with complicated geometries (such as optical metamaterials with plasmonic regions, which are commonly modeled with volume formulations in the literature).

Section 2 contains details of the implementation, including formulations with JMCFIE, discretization, and iterative solutions via parallel MLFMA. Numerical examples are presented in Section 3 , followed by our concluding remarks in Section $\underline{4}$. 


\section{PARALLEL IMPLEMENTATION OF MLFMA}

We consider the solution of electromagnetics problems involving composite objects with multiple dielectric and/or metallic parts. Let the three-dimensional space $D$ be divided into $U+1$ homogeneous regions as

$$
D=\bigcup_{u=0}^{U} D_{u}
$$

where $D_{0}$ is the background medium (free space in this paper) containing other regions and extending to infinity. A region $D_{u}$ for $u \neq 0$ may consist of multiple unconnected bodies with the same material properties. Time-harmonic electromagnetic sources with $e^{-i \omega t}$ time dependence are used for excitation.

\section{A. Surface Integral Equations}

Consider a homogeneous region $D_{u}$ that is characterized by a complex permittivity $\epsilon_{u}=\epsilon_{0} \epsilon_{r, u}$ and a complex permeability $\mu_{u}=\mu_{0} \mu_{r, u}$, where $\epsilon_{r, u}$ and $\mu_{r, u}$ are the relative permittivity and permeability, respectively. The integro-differential operators associated with this region can be written as

$$
\begin{aligned}
\mathcal{T}_{u}\{\boldsymbol{X}\}(\mathbf{r})= & i k_{u} \int_{S_{u}} \mathrm{~d} \boldsymbol{r}^{\prime} \boldsymbol{X}\left(\boldsymbol{r}^{\prime}\right) g_{u}\left(\boldsymbol{r}, \boldsymbol{r}^{\prime}\right) \\
& +\frac{i}{k_{u}} \int_{S_{u}} \mathrm{~d} \boldsymbol{r}^{\prime} \nabla^{\prime} \cdot \boldsymbol{X}\left(\boldsymbol{r}^{\prime}\right) \nabla g_{u}\left(\boldsymbol{r}, \boldsymbol{r}^{\prime}\right), \\
\mathcal{K}_{u}^{\mathrm{PV}}\{\boldsymbol{X}\}(\boldsymbol{r})= & \int_{\mathrm{PV}, S_{u}} \mathrm{~d} \boldsymbol{r}^{\prime} \boldsymbol{X}\left(\boldsymbol{r}^{\prime}\right) \times \nabla^{\prime} g_{u}\left(\boldsymbol{r}, \boldsymbol{r}^{\prime}\right),
\end{aligned}
$$

where PV indicates the principal value of the integral, $S_{u}$ is the surface or a set of surfaces bounding the region (that will be called "the surface of the region" in the rest of the paper),

$$
g_{u}\left(\boldsymbol{r}, \boldsymbol{r}^{\prime}\right)=\frac{\exp \left(i k_{u}\left|\boldsymbol{r}-\boldsymbol{r}^{\prime}\right|\right)}{4 \pi\left|\boldsymbol{r}-\boldsymbol{r}^{\prime}\right|}
$$

denotes the homogeneous-space Green's function, and

$$
k_{u}=\omega \sqrt{\epsilon_{u}} \sqrt{\mu_{u}}=\frac{2 \pi}{\lambda_{u}}
$$

is the wavenumber. Testing the boundary condition for the electric field on the surface of the region, tangential and normal forms of the electric-field integral equation (T-EFIE and $\mathrm{N}-\mathrm{EFIE}$ ) are derived as

$$
\begin{aligned}
& \left\{\begin{array}{c}
-\hat{\boldsymbol{n}} \times \hat{\boldsymbol{n}} \times \\
\hat{\boldsymbol{n}} \times
\end{array}\right\}\left\{\eta_{u} \mathcal{T}_{u}\{\boldsymbol{J}\}-\mathcal{K}_{u}^{\mathrm{PV}}\{\boldsymbol{M}\}-\frac{\Omega_{u}}{4 \pi} \hat{\boldsymbol{n}} \times \boldsymbol{M}\right\}(\boldsymbol{r}) \\
& =-\left\{\begin{array}{c}
-\hat{\boldsymbol{n}} \times \hat{\boldsymbol{n}} \times \\
\hat{\boldsymbol{n}} \times
\end{array}\right\} \boldsymbol{E}_{u}^{\mathrm{inc}}(\boldsymbol{r}),
\end{aligned}
$$

where $\boldsymbol{E}_{u}^{\mathrm{inc}}$ is the incident electric field created by external sources located inside the region. According to this conventional naming scheme [25], the terms "tangential" and "normal" describe the testing strategy rather than the tested component. Even though only the tangential component is tested in both cases, it is tested directly and rotationally in the tangential and normal integral equations, respectively.

Similarly, testing the boundary condition for the magnetic field, tangential and normal forms of the magnetic-field integral equation (T-MFIE and N-MFIE) are derived as

$$
\begin{aligned}
& \left\{\begin{array}{c}
-\hat{\boldsymbol{n}} \times \hat{\boldsymbol{n}} \times \\
\hat{\boldsymbol{n}} \times
\end{array}\right\}\left\{\eta_{u}^{-1} \mathcal{T}_{u}\{\boldsymbol{M}\}+\mathcal{K}_{u}^{\mathrm{PV}}\{\boldsymbol{J}\}+\frac{\Omega_{u}}{4 \pi} \hat{\boldsymbol{n}} \times \boldsymbol{J}\right\}(\boldsymbol{r}) \\
& =-\left\{\begin{array}{c}
-\hat{\boldsymbol{n}} \times \hat{\boldsymbol{n}} \times \\
\hat{\boldsymbol{n}} \times
\end{array}\right\} \boldsymbol{H}_{u}^{\mathrm{inc}}(\boldsymbol{r}),
\end{aligned}
$$

where $\boldsymbol{H}_{u}^{\text {inc }}$ is the incident magnetic field created by external sources located inside the region. In (6) and (7), $r \in S_{u}$ is the observation point, $\hat{\boldsymbol{n}}$ is the oriented unit normal vector, $\Omega_{u} \in$ $(0,4 \pi)$ is the solid angle, $\eta_{u}=\sqrt{\mu_{u}} / \sqrt{\epsilon_{u}}$ is the intrinsic impedance of the region, and $\boldsymbol{J}=\hat{\boldsymbol{n}} \times \boldsymbol{H}$ and $\boldsymbol{M}=-\hat{\boldsymbol{n}} \times \boldsymbol{E}$ are the equivalent electric and magnetic currents, respectively. If the observation point is on a PEC surface, the magnetic current $(\boldsymbol{M})$ is set to zero.

\section{B. Formulation with JMCFIE}

For a given problem, the basic integral equations, namely, T-EFIE, N-EFIE, T-MFIE, and N-MFIE defined in Eqs. (ㅁ) and (7), can be combined in many ways to derive different formulations. Among various formulations, JMCFIE is worth noting as a stable formulation that is free of internal resonances and suitable for composite structures [24-30]. JMCFIE can be written as

$$
\sum_{u=0}^{U}\left[\begin{array}{c}
\eta_{u}^{-1} \alpha \mathrm{T}-\operatorname{EFIE}(u)+(1-\alpha) \mathrm{N}-\operatorname{MFIE}(u) \\
\eta_{u} \alpha \mathrm{T}-\operatorname{MrIE}(u)-(1-\alpha) \mathrm{N}-\operatorname{EFIE}(u)
\end{array}\right],
$$

where $\alpha \in[0,1]$ is a combination parameter that is set to 0.5 in this paper. Depending on the problem and its discretization, the choice of this parameter can be critical in terms of accuracy and efficiency since it defines the relative contributions of the tangential and normal integral equations in JMCFIE. Favorable properties of JMCFIE over other integral-equation formulations for penetrable objects, including those with negative permittivity and permeability, are extensively discussed in $[\underline{26}, \underline{29}, \underline{30}]$.

\section{Discretization of JMCFIE}

Simultaneous discretizations of JMCFIE and surfaces using a set of RWG functions on planar triangles lead to $2 N \times 2 N$ matrix equations in the form of

$$
\left[\begin{array}{ll}
\overline{\boldsymbol{Z}}^{(11)} & \overline{\boldsymbol{Z}}^{(12)} \\
\overline{\boldsymbol{Z}}^{(21)} & \overline{\boldsymbol{Z}}^{(22)}
\end{array}\right] \cdot\left[\begin{array}{l}
\boldsymbol{a}^{(1)} \\
\boldsymbol{a}^{(2)}
\end{array}\right]=\left[\begin{array}{l}
\boldsymbol{v}^{(1)} \\
\boldsymbol{v}^{(2)}
\end{array}\right]
$$

where

$$
\left[\begin{array}{ll}
\overline{\boldsymbol{Z}}^{(11)} & \overline{\boldsymbol{Z}}^{(12)} \\
\overline{\boldsymbol{Z}}^{(21)} & \overline{\boldsymbol{Z}}^{(22)}
\end{array}\right]=\sum_{u=0}^{U}\left[\begin{array}{ll}
\overline{\boldsymbol{Z}}_{u}^{(11)} & \overline{\boldsymbol{Z}}_{u}^{(12)} \\
\overline{\boldsymbol{Z}}_{u}^{(21)} & \overline{\boldsymbol{Z}}_{u}^{(22)}
\end{array}\right]
$$

and

$$
\left[\begin{array}{l}
\boldsymbol{v}^{(1)} \\
\boldsymbol{v}^{(2)}
\end{array}\right]=\sum_{u=0}^{U}\left[\begin{array}{l}
\boldsymbol{v}_{u}^{(1)} \\
\boldsymbol{v}_{u}^{(2)}
\end{array}\right]
$$


Matrix elements in (10) can be interpreted as electromagnetic interactions between discretization elements, i.e., basis and testing functions.

Consider the $n$th basis function $\boldsymbol{b}_{n}$ and the $m$ th testing function $t_{m}$. Their interactions through a penetrable region $D_{u}$ can be nonzero only if they are located partially or entirely on the surface of the region. Then,

$$
\begin{aligned}
\overline{\boldsymbol{Z}}_{u}^{(11)}[m, n]= & \alpha \overline{\boldsymbol{T}}_{u}^{T}[m, n]+(1-\alpha) \overline{\boldsymbol{K}}_{u}^{\mathrm{PV}, N}[m, n] \\
& -\frac{1}{2}(1-\alpha) \overline{\boldsymbol{I}}^{T}[m, n] .
\end{aligned}
$$

Also, if the basis function is not located on a PEC surface,

$$
\overline{\boldsymbol{Z}}_{u}^{(12)}[m, n]=\eta_{u}^{-1}\left\{(1-\alpha) \overline{\boldsymbol{T}}_{u}^{N}[m, n]-\alpha \overline{\boldsymbol{K}}_{u}^{\mathrm{PV}, T}[m, n]-\frac{1}{2} \alpha \overline{\boldsymbol{I}}^{N}[m, n]\right\} .
$$

Similarly, if the testing function is not located on a PEC surface,

$$
\overline{\mathbf{Z}}_{u}^{(21)}[m, n]=-\eta_{u}^{2} \overline{\mathbf{Z}}_{u}^{(12)}[m, n]
$$

Finally, if both of the basis and testing functions are not located on PEC surfaces,

$$
\overline{\mathbf{Z}}_{u}^{(22)}[m, n]=\overline{\mathbf{Z}}_{u}^{(11)}[m, n] .
$$

Basis and testing functions located on PEC surfaces lead to zero matrix elements in some of the partitions. For nonzero interactions associated with a region $D_{u}$, the discretized operators are defined as

$$
\begin{aligned}
\overline{\boldsymbol{T}}_{u}^{T}[m, n] & =\gamma_{u n} \gamma_{u m} \int_{S_{u m}} \mathrm{~d} \boldsymbol{r} \boldsymbol{t}_{m}(\boldsymbol{r}) \cdot \mathcal{T}_{u}\left\{\boldsymbol{b}_{n}\right\}(\boldsymbol{r}), \\
\overline{\boldsymbol{T}}_{u}^{N}[m, n] & =\gamma_{u n} \int_{S_{u m}} \mathrm{~d} \boldsymbol{r} \boldsymbol{t}_{m}^{\times n}(\boldsymbol{r}) \cdot \mathcal{T}_{u}\left\{\boldsymbol{b}_{n}\right\}(\boldsymbol{r}), \\
\overline{\boldsymbol{K}}_{u}^{\mathrm{PV}, T}[m, n] & =\gamma_{u n} \gamma_{u m} \int_{S_{u m}} \mathrm{~d} \boldsymbol{r} \boldsymbol{t}_{m}(\boldsymbol{r}) \cdot \mathcal{K}_{u}^{\mathrm{PV}}\left\{\boldsymbol{b}_{n}\right\}(\boldsymbol{r}), \\
\overline{\boldsymbol{K}}_{u}^{\mathrm{PV}, N}[m, n] & =\gamma_{u n} \int_{S_{u m}} \mathrm{~d} \boldsymbol{r} \boldsymbol{t}_{m}^{\times n}(\boldsymbol{r}) \cdot \mathcal{K}_{u}^{\mathrm{PV}}\left\{\boldsymbol{b}_{n}\right\}(\boldsymbol{r}), \\
\overline{\boldsymbol{I}}_{u}^{T}[m, n] & =\gamma_{u n} \gamma_{u m} \int_{S_{u m}} \mathrm{~d} \boldsymbol{r} \boldsymbol{t}_{m}(\boldsymbol{r}) \cdot \boldsymbol{b}_{n}(\boldsymbol{r}), \\
\overline{\boldsymbol{I}}_{u}^{N}[m, n] & =\gamma_{u n} \int_{S_{u m}} \mathrm{~d} \boldsymbol{r} \boldsymbol{t}_{m}^{\times n}(\boldsymbol{r}) \cdot \boldsymbol{b}_{n}(\boldsymbol{r}),
\end{aligned}
$$

where $S_{u m}$ is the spatial support of the $m$ th testing function on the surface of $D_{u}, \boldsymbol{t}_{m}^{\times n}=\boldsymbol{t}_{m} \times \hat{\boldsymbol{n}}$, and $\gamma_{u n}= \pm 1$ and $\gamma_{u m}= \pm 1$ represent the orientations of the basis and testing functions with respect to the surface of the region [32].
Let external sources exist in a penetrable region $D_{u}$. The incident electromagnetic fields created by these sources are tested by the $m$ th testing function (that is located on the surface of the region) as

$$
\begin{aligned}
\boldsymbol{v}_{u}^{(1)}[m]= & -\eta_{u}^{-1} \alpha \gamma_{u m} \int_{S_{u m}} \mathrm{~d} \boldsymbol{r} \boldsymbol{t}_{m}(\boldsymbol{r}) \cdot \boldsymbol{E}_{u}^{\mathrm{inc}}(\boldsymbol{r}) \\
& -(1-\alpha) \int_{S_{u m}} \mathrm{~d} \boldsymbol{r} \boldsymbol{t}_{m}^{\times n}(\boldsymbol{r}) \cdot \boldsymbol{H}_{u}^{\mathrm{inc}}(\boldsymbol{r}) .
\end{aligned}
$$

If the testing function is not located on a PEC surface,

$$
\begin{aligned}
\boldsymbol{v}_{u}^{(2)}[m]= & (1-\alpha) \int_{S_{u m}} \mathrm{~d} \boldsymbol{r} \boldsymbol{t}_{m}^{\times n}(\boldsymbol{r}) \cdot \boldsymbol{E}_{u}^{\mathrm{inc}}(\boldsymbol{r}) \\
& -\eta_{u} \alpha \gamma_{u m} \int_{S_{u m}} \mathrm{~d} \boldsymbol{r} \boldsymbol{t}_{m}(\boldsymbol{r}) \cdot \boldsymbol{H}_{u}^{\mathrm{inc}}(\boldsymbol{r}) .
\end{aligned}
$$

As a common practice, in the host region $D_{0}$ that extends to infinity, incident fields can be defined as plane waves without any source region.

\section{Iterative Solutions with MLFMA}

Matrix equations derived from JMCFIE can be solved iteratively, where the required matrix-vector multiplications are performed as

$$
\begin{aligned}
{\left[\begin{array}{l}
\boldsymbol{y}^{(1)} \\
\boldsymbol{y}^{(2)}
\end{array}\right]=} & \sum_{u=0}^{U}\left[\begin{array}{ll}
\overline{\boldsymbol{Z}}_{u}^{(11)} & \overline{\boldsymbol{Z}}_{u}^{(12)} \\
\overline{\boldsymbol{Z}}_{u}^{(21)} & \overline{\boldsymbol{Z}}_{u}^{(22)}
\end{array}\right] \cdot\left[\begin{array}{l}
\boldsymbol{x}^{(1)} \\
\boldsymbol{x}^{(2)}
\end{array}\right] \\
= & \sum_{u=0}^{U}\left[\begin{array}{ll}
\overline{\boldsymbol{Z}}_{\mathrm{NF}, u}^{(11)} & \overline{\boldsymbol{Z}}_{\mathrm{NF}, u}^{(12)} \\
\overline{\boldsymbol{Z}}_{\mathrm{NF}, u}^{(21)} & \overline{\boldsymbol{Z}}_{\mathrm{NF}, u}^{(22)}
\end{array}\right] \cdot\left[\begin{array}{l}
\boldsymbol{x}^{(1)} \\
\boldsymbol{x}^{(2)}
\end{array}\right] \\
& +\sum_{u=0}^{U}\left[\begin{array}{ll}
\overline{\boldsymbol{Z}}_{\mathrm{FF}, u}^{(11)} & \overline{\boldsymbol{Z}}_{\mathrm{FF}, u}^{(12)} \\
\overline{\boldsymbol{Z}}_{\mathrm{FF}, u}^{(21)} & \overline{\boldsymbol{Z}}_{\mathrm{FF}, u}^{(22)}
\end{array}\right] \cdot\left[\begin{array}{l}
\boldsymbol{x}^{(1)} \\
\boldsymbol{x}^{(2)}
\end{array}\right] .
\end{aligned}
$$

In (25), $\overline{\boldsymbol{Z}}_{\mathrm{NF}, u}^{(a b)}$ and $\overline{\boldsymbol{Z}}_{\mathrm{FF}, u}^{(a b)}$ for $a=1,2$ and $b=1,2$ represent near-field and far-field interactions, respectively, associated with a region $D_{u}$. We use the one-box-buffer scheme [33] to define near-field and far-field interactions. Near-field interactions, which are between closely located basis and testing functions, are combined in a single sparse matrix with $\mathcal{O}(N)$ nonzero elements. Hence, (25) can be rewritten as

$$
\begin{aligned}
{\left[\begin{array}{l}
\boldsymbol{y}^{(1)} \\
\boldsymbol{y}^{(2)}
\end{array}\right]=} & {\left[\begin{array}{ll}
\overline{\boldsymbol{Z}}_{\mathrm{NF}}^{(11)} & \overline{\boldsymbol{Z}}_{\mathrm{NF}}^{(12)} \\
\overline{\boldsymbol{Z}}_{\mathrm{NF}}^{(21)} & \overline{\boldsymbol{Z}}_{\mathrm{NF}}^{(22)}
\end{array}\right] \cdot\left[\begin{array}{c}
\boldsymbol{x}^{(1)} \\
\boldsymbol{x}^{(2)}
\end{array}\right] } \\
& +\sum_{u=0}^{U}\left[\begin{array}{ll}
\overline{\boldsymbol{Z}}_{\mathrm{FF}, u}^{(11)} & \overline{\boldsymbol{Z}}_{\mathrm{FF}, u}^{(12)} \\
\overline{\boldsymbol{Z}}_{\mathrm{FF}, u}^{(21)} & \overline{\boldsymbol{Z}}_{\mathrm{FF}, u}^{(22)}
\end{array}\right] \cdot\left[\begin{array}{l}
\boldsymbol{x}^{(1)} \\
\boldsymbol{x}^{(2)}
\end{array}\right],
\end{aligned}
$$

where $\overline{\boldsymbol{Z}}_{\mathrm{NF}}^{(a b)}$ for $a=1,2$ are calculated directly and stored in memory to be used multiple times during an iterative solution. On the other hand, far-field interactions are performed efficiently using the factorization and diagonalization of the Green's function, as described in the next subsection.

\section{E. Far-Field Interactions}

For each penetrable region $D_{u}$, a tree structure of $L_{u}$ levels is constructed by placing the surface of the region in a cubic box and recursively dividing it into subboxes. If $L_{u} \geq 3$, a sequence of aggregation, translation, and disaggregation stages 
is carried out for each matrix-vector multiplication in the form of

$$
\boldsymbol{y}^{(a)} \leftarrow \overline{\boldsymbol{Z}}_{\mathrm{FF}, u}^{(a b)} \cdot \boldsymbol{x}^{(b)}
$$

for $a=1,2$ and $b=1,2$.

The aggregation stage is performed from the lowest level $(l=1)$ to the highest level involving translations, i.e., $l=L_{u}-2$. The radiated field of a box $C$ at the lowest level with respect to a reference point $\boldsymbol{r}_{C}$ is calculated as

$$
\boldsymbol{S}_{u C}^{(b)}\left(\hat{\boldsymbol{k}}, \boldsymbol{r}_{C}\right)=\sum_{\boldsymbol{b}_{n} \in C} \boldsymbol{S}_{u n}\left(\hat{\boldsymbol{k}}, \boldsymbol{r}_{C}\right) \boldsymbol{x}^{(b)}[n],
$$

where the radiation pattern of the $n$th basis function is defined as

$$
\boldsymbol{S}_{u n}\left(\hat{\boldsymbol{k}}, \boldsymbol{r}_{C}\right)=\left(\overline{\boldsymbol{I}}_{3 \times 3}-\hat{\boldsymbol{k}} \hat{\boldsymbol{k}}\right) \cdot \gamma_{u n} \boldsymbol{R}_{u n}^{-}\left(\hat{\boldsymbol{k}}, \boldsymbol{r}_{C}\right) .
$$

In (29), $\overline{\boldsymbol{I}}_{3 \times 3}$ is the $3 \times 3$ unit dyad and

$$
\boldsymbol{R}_{u n}^{-}\left(\hat{\boldsymbol{k}}, \boldsymbol{r}_{C}\right)=\int_{S_{u n}} \mathrm{~d} \boldsymbol{r} \exp \left[-i k_{u} \hat{\boldsymbol{k}} \cdot\left(\boldsymbol{r}-\boldsymbol{r}_{C}\right)\right] \boldsymbol{b}_{n}(\boldsymbol{r})
$$

can be interpreted as a Fourier transform, where $S_{u n}$ is the spatial support of the basis function on the surface of $D_{u}$. Note that, if $b=2$, basis functions on PEC surfaces have no contribution in (28). For a box $C$ at a higher level,

$$
\boldsymbol{S}_{u C}^{(b)}\left(\hat{\boldsymbol{k}}, \boldsymbol{r}_{C}\right)=\sum_{C^{\prime} \in C} \beta_{u}\left(\hat{\boldsymbol{k}}, \boldsymbol{r}_{C}-\boldsymbol{r}_{C^{\prime}}\right) \boldsymbol{S}_{u C^{\prime}}^{(b)}\left(\hat{\boldsymbol{k}}, \boldsymbol{r}_{C^{\prime}}\right)
$$

where

$$
\beta_{u}(\hat{\boldsymbol{k}}, \boldsymbol{r})=\exp \left(i k_{u} \hat{\boldsymbol{k}} \cdot \boldsymbol{r}\right)
$$

is the diagonal shift operator.

In the translation stage, radiated fields are converted into incoming fields between boxes. The incoming field for a box $C$ is defined as

$$
\boldsymbol{G}_{u C}^{(b)}\left(\hat{\boldsymbol{k}}, \boldsymbol{r}_{C}\right)=\sum_{C^{\prime} \in F\{C\}} \tau_{u}\left(\hat{\boldsymbol{k}}, \boldsymbol{r}_{C}-\boldsymbol{r}_{C^{\prime}}\right) \boldsymbol{S}_{u C^{\prime}}^{(b)}\left(\hat{\boldsymbol{k}}, \boldsymbol{r}_{C^{\prime}}\right),
$$

where $F\{C\}$ represents the far-zone list of $C$, and

$$
\tau_{u}(\hat{\boldsymbol{k}}, \boldsymbol{r})=\sum_{t=0}^{T_{u}} i^{t}(2 t+1) h_{t}^{(1)}\left(k_{u} r\right) P_{t}(\hat{\boldsymbol{k}} \cdot \hat{\boldsymbol{r}})
$$

is the diagonal translation operator [34] for $\boldsymbol{r}=r \hat{\boldsymbol{r}}$. In (34), $h_{t}^{(1)}$ is the spherical Hankel function of the first kind, $P_{t}$ is the Legendre polynomial, and $T_{u}$ is the number of terms that can be determined by the excess bandwidth formula [3,33].

In the disaggregation stage, total incoming fields are calculated from level $l=L_{u}-2$ to $l=1$. The total incoming field for a box $C$ is the sum of incoming fields due to translations and the incoming field from its parent box, i.e.,

$$
\tilde{\boldsymbol{G}}_{u C}^{(b)}\left(\hat{\boldsymbol{k}}, \boldsymbol{r}_{C}\right)=\boldsymbol{G}_{u C}^{(b)}\left(\hat{\boldsymbol{k}}, \boldsymbol{r}_{C}\right)+\beta_{u}\left(\hat{\boldsymbol{k}}, \boldsymbol{r}_{C}-\boldsymbol{r}_{C^{\prime}}\right) \tilde{\boldsymbol{G}}_{u C^{\prime}}^{(b)}\left(\hat{\boldsymbol{k}}, \boldsymbol{r}_{C^{\prime}}\right),
$$

where $C \in C^{\prime}$. At the lowest level, total incoming fields are received by testing functions as

$$
\sum_{n=1}^{N} \overline{\boldsymbol{Z}}_{\mathrm{FF}, u}^{(a b)}[m, n] \boldsymbol{x}^{(b)}[n] \approx\left(\frac{i \boldsymbol{k}_{u}}{4 \pi}\right)^{2} \int d^{2} \hat{\boldsymbol{k}} \boldsymbol{F}_{u m}^{(a b)}\left(\hat{\boldsymbol{k}}, \boldsymbol{r}_{C}\right) \cdot \tilde{\boldsymbol{G}}_{u C}^{(b)}\left(\hat{\boldsymbol{k}}, \boldsymbol{r}_{C}\right)
$$

for $\boldsymbol{t}_{m} \in C$. Note that, if $a=2$, the value of (36) is set to zero for testing functions on PEC surfaces. If (36) is nonzero, the receiving pattern of the $m$ th testing function depends on the partition, i.e.,

$$
\begin{aligned}
\boldsymbol{F}_{u m}^{(11)}\left(\hat{\boldsymbol{k}}, \boldsymbol{r}_{C}\right)= & \alpha\left(\overline{\boldsymbol{I}}_{3 \times 3}-\hat{\boldsymbol{k}} \hat{\boldsymbol{k}}\right) \cdot \gamma_{u m} \boldsymbol{R}_{u m}^{+}\left(\hat{\boldsymbol{k}}, \boldsymbol{r}_{C}\right) \\
& -(1-\alpha) \hat{\boldsymbol{k}} \times \boldsymbol{R}_{u m}^{\times n}\left(\hat{\boldsymbol{k}}, \boldsymbol{r}_{C}\right),
\end{aligned}
$$

$$
\begin{aligned}
\boldsymbol{F}_{u m}^{(12)}\left(\hat{\boldsymbol{k}}, \boldsymbol{r}_{C}\right)= & \eta_{u}^{-1}\left\{(1-\alpha)\left(\overline{\boldsymbol{I}}_{3 \times 3}-\hat{\boldsymbol{k}} \hat{\boldsymbol{k}}\right) \cdot \boldsymbol{R}_{u m}^{\times n}\left(\hat{\boldsymbol{k}}, \boldsymbol{r}_{C}\right)\right. \\
& \left.+\alpha \gamma_{u m} \hat{\boldsymbol{k}} \times \boldsymbol{R}_{u m}^{+}\left(\hat{\boldsymbol{k}}, \boldsymbol{r}_{C}\right)\right\},
\end{aligned}
$$

$\boldsymbol{F}_{u m}^{(21)}\left(\hat{\boldsymbol{k}}, \boldsymbol{r}_{C}\right)=-\eta_{u}^{2} \boldsymbol{F}_{u m}^{(12)}\left(\hat{\boldsymbol{k}}, \boldsymbol{r}_{C}\right)$,

$$
\boldsymbol{F}_{u m}^{(22)}\left(\hat{\boldsymbol{k}}, \boldsymbol{r}_{C}\right)=\boldsymbol{F}_{u m}^{(11)}\left(\hat{\boldsymbol{k}}, \boldsymbol{r}_{C}\right),
$$

where

$$
\begin{aligned}
& \boldsymbol{R}_{u m}^{+}\left(\hat{\boldsymbol{k}}, \boldsymbol{r}_{C}\right)=\int_{S_{u m}} \mathrm{~d} \boldsymbol{r} \exp \left[i k_{u} \hat{\boldsymbol{k}} \cdot\left(\boldsymbol{r}-\boldsymbol{r}_{C}\right)\right] \boldsymbol{t}_{m}(\boldsymbol{r}), \\
& \boldsymbol{R}_{u m}^{\times n}\left(\hat{\boldsymbol{k}}, \boldsymbol{r}_{C}\right)=\int_{S_{u m}} \mathrm{~d} \boldsymbol{r} \exp \left[i k_{u} \hat{\boldsymbol{k}} \cdot\left(\boldsymbol{r}-\boldsymbol{r}_{C}\right)\right] \boldsymbol{t}_{m}^{\times n}(\boldsymbol{r}) .
\end{aligned}
$$

For real values of $k_{u}$ and using a Galerkin discretization with real-valued basis and testing functions,

$$
\boldsymbol{R}_{u m}^{+}\left(\hat{\boldsymbol{k}}, \boldsymbol{r}_{C}\right)=\left(\boldsymbol{R}_{u m}^{-}\left(\hat{\boldsymbol{k}}, \boldsymbol{r}_{C}\right)\right)^{*},
$$

where “*” represents the complex-conjugate operation.

\section{F. Sampling and Interpolation}

Consider a tree structure of $L_{u}$ levels associated with a region $D_{u}$. At level $l=1,2, L_{u}-2$, radiated and incoming fields are sampled at $S_{u l}=\mathcal{O}\left(k_{u}^{2} a_{l}^{2}\right)$ angular directions, where $a_{l}$ is the box size. We choose samples regularly spaced in the $\phi$ direction and apply the Gauss-Legendre quadrature in the $\theta$ direction [34]. Note that radiation and receiving patterns of basis and testing functions are sampled according to the lowestlevel sampling scheme and they are stored in memory to be used multiple times during iterations. Translation operators at all levels are also sampled and stored in memory before iterative solutions.

Since the sampling rate depends on the box size as measured by wavelength, radiated and incoming fields are sampled at diverse rates from level to level of a tree structure. Hence, interpolations are required during the aggregation and disaggregation stages to match the different sampling rates of consecutive levels. We employ a sequence of 1-D local Lagrange interpolation methods [35], leading to $\mathcal{O}(1)$ computational complexity per sample. As commonly practiced in the literature, interpolations during the disaggregation stage are 
replaced with adjoint interpolations (anterpolations) to improve the accuracy without sacrificing the efficiency [36].

If the surface of the region $D_{u}$ is discretized with a total of $N_{u}$ unknowns, the associated tree structure has $\mathcal{O}\left(\log N_{u}\right)$ levels. The time and memory costs of MLFMA, which correspond to the total number of field samples, are $\mathcal{O}\left(N_{u}\right)$ per level, leading to $\mathcal{O}\left(N_{u} \log N_{u}\right)$ overall complexity.

\section{G. Parallelization}

Consider the parallelization of a tree structure associated with a region $D_{u}$. At level $l=1,2, L_{u}-2$, there are $N_{u l}$ boxes and $S_{u l}$ samples per box. In general, the number of boxes and the number samples balance each other, i.e., the cost per level $\left(N_{u l} S_{u l}\right)$ is almost constant and it is $\mathcal{O}\left(N_{u}\right)$ independent of the index of the level. Let the tree structure be parallelized into $P$ processes. At level $l$, a process $p \in\{1,2, \ldots, P\}$ is assigned to $N_{u l}^{p}$ boxes and $S_{u l}^{p}$ samples. Using the hierarchical strategy, $N_{u l}^{p} \approx N_{u l} / P_{N}$ and $S_{u l}^{p} \approx S_{u l} / P_{S}$, where $P_{N}$ and $P_{S}=$ $P / P_{N}$ are determined by load-balancing algorithms. For easier implementations, we assume that the numbers of boxes and sample partitions (hence the number of processes) are powers of two, i.e., $P_{N}=2^{v}, P_{S}=2^{w}$, and $P=2^{v+w}$ for $v, w \geq 0$.

In order to facilitate operations in the aggregation stage, intermediate levels $l+1 / 2$ are defined for $l=1,2, \ldots, L_{l}-3$. For an aggregation from level $l$ to level $l+1$, locally available boxes at the intermediate level $l+1 / 2$ are traced one by one. If field samples are partitioned at the lower level $(l)$, interpolations may require exchange of samples between processes. These are called vertical communications and they are between neighboring processes that are assigned to different samples of the same set of boxes. Following each vertical communication, the inflated data is interpolated and shifted as usual to compute the radiated field of the parent box at the intermediate level $(l+1 / 2)$. After all boxes at the intermediate level are considered, data exchanges, namely diagonal communications, may be required between pairs of processes if the partitioning of boxes and field samples is different at the higher level $(l+1)$. Note that intermediate levels are defined particularly to prepare and store data before these data exchanges (see [13] for a detailed discussion on intermediate levels).

During the translation stage, intraprocess translations that are between locally available boxes are performed in each process. These translations are followed by interprocess translations, which are between those boxes located in different processes so that communications are required. Specifically, each process is paired with $P_{N}$ processes to perform interprocess translations via communications. These are called horizontal communications and they are between those processes (not necessarily neighboring) that are assigned to the same set of samples of different boxes. Once a pairing is established, all levels and boxes at these levels are traced to transfer the required radiated fields from the sender process to the receiver process and converting them into incoming fields on the receiver side.

Parallelization of the disaggregation stage is very similar to but reverse of that of the aggregation stage. After diagonal communications take place to obtain data for an intermediate level, incoming fields are shifted and anterpolated. For each box at the lower level, samples obtained by an anterpolation operation are deflated via vertical communications and superposed with the corresponding samples of incoming fields due to translations. At the end of the disaggregation stage, each process performs receiving operations as defined in (36) using the locally available data at the lowest level to obtain a part of the output vector.

\section{NUMERICAL EXAMPLES}

In this section, we present electromagnetic simulations involving large-scale composite objects. For numerical solutions, the parallel implementation detailed in Section 2 is employed on 64 processes of a cluster of Intel Xeon Nehalem processors with $2.80 \mathrm{GHz}$ clock rate. Matrix-vector multiplications are performed by MLFMA with two digits of accuracy, i.e., all matrix elements are computed with maximum $1 \%$ error [18]. Iterative solutions are performed by the biconjugategradient-stabilized (BiCGStab) algorithm [37] accelerated with block-diagonal preconditioners [26], and the target residual error for the convergence is set to 0.005 .

Figure 1 presents solutions of electromagnetics problems involving a spherical composite object. A metallic sphere of radius $50 \mu \mathrm{m}$ (core) is placed inside a dielectric sphere of radius $100 \mu \mathrm{m}$ (shell). The core is modeled as a PEC, whereas the shell is lossless dielectric with a relative permittivity of 2 . The object is illuminated by a plane wave propagating in the $z$ direction with the electric field polarized in the
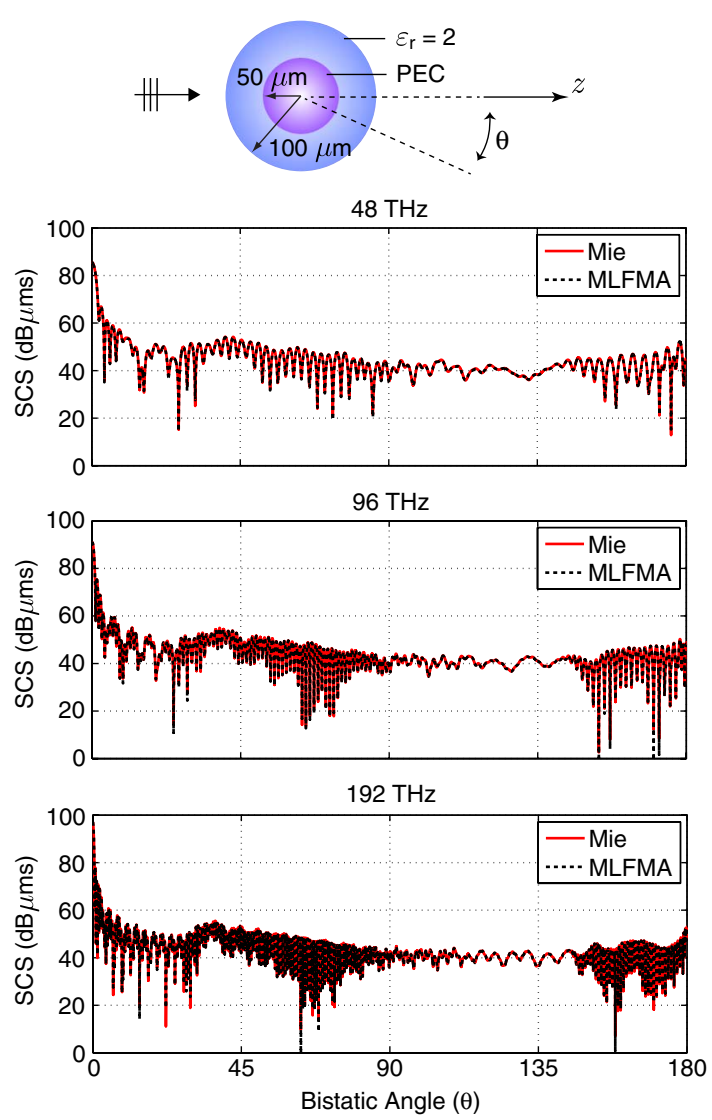

Fig. 1. (Color online) Solutions of electromagnetics problems involving a spherical composite object illuminated by a plane wave at three different frequencies, i.e., 48, 96, and $192 \mathrm{THz}$. At each frequency, SCS is plotted with respect to the bistatic angle from $0^{\circ}$ to $180^{\circ}$, where $0^{\circ}$ and $180^{\circ}$ correspond to the forward-scattering and backscattering directions, respectively. 
Table 1. Solutions of Electromagnetics Problems Involving a Spherical Composite Object Depicted in Fig. 1

\begin{tabular}{lccc}
\hline Frequency (THz) & 48 & 96 & 192 \\
Unknowns & $3,278,208$ & $13,112,832$ & $52,451,328$ \\
Iterations & 51 & 44 & 48 \\
Total time (h) & 0.90 & 3.44 & 14.7 \\
Peak memory (GB) & 24.7 & 97.4 & 389 \\
RMS error & $0.65 \%$ & $0.64 \%$ & $0.57 \%$ \\
\hline
\end{tabular}

$x$ direction at three different frequencies, i.e., 48, 96, and $192 \mathrm{THz}$. At these frequencies, discretizations of the object with the RWG functions on $\lambda_{0} / 10$ triangles lead to matrix equations involving 3,278,208, 13,112,832, and 52,451,328 unknowns, respectively. Figure 1 depicts the bistatic scattering cross section (SCS) values in $\mathrm{dB} \mu \mathrm{ms}$ on the $z-x$ plane with respect to the bistatic angle from $0^{\circ}$ (forward-scattering direction) to $180^{\circ}$ (backscattering direction). Computational values obtained by using MLFMA are compared with those obtained via analytical Mie-series solutions. It can be observed that the computational values agree very well with the analytical results. Table 1 lists the number of iterations, total time (including setup and iterative solutions), total peak memory, and root-mean-square (RMS) error in the computational SCS values with respect to the analytical values. The RMS
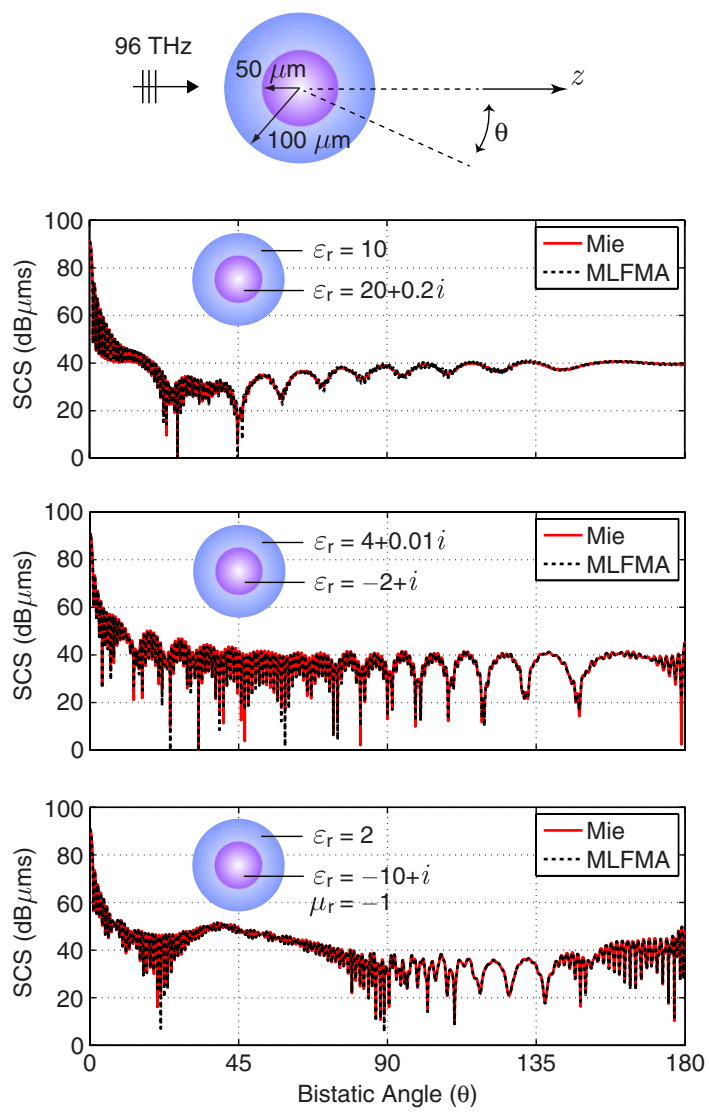

Fig. 2. (Color online) Solutions of electromagnetics problems involving spherical composite objects, each of which is illuminated by a plane wave at $96 \mathrm{THz}$. SCS is plotted with respect to the bistatic angle from $0^{\circ}$ to $180^{\circ}$, where $0^{\circ}$ and $180^{\circ}$ correspond to the forwardscattering and backscattering directions, respectively.
Table 2. Solutions of Electromagnetics Problems Involving Spherical Composite Objects Depicted in Fig. $\underline{2}$

\begin{tabular}{lccc}
\hline$\epsilon_{r}$ (Shell) & 10 & $4+0.01 i$ & 2 \\
$\epsilon_{r}$ (Core) & $20+0.2 i$ & $-2+i$ & $-10+i$ \\
$\mu_{r}$ (Core) & 1 & 1 & -1 \\
Iterations & 58 & 24 & 48 \\
Total time (hours) & 15.6 & 3.83 & 7.01 \\
Peak memory (GB) & 587 & 207 & 351 \\
RMS error & $0.52 \%$ & $0.64 \%$ & $0.51 \%$ \\
\hline
\end{tabular}

error is less than the target $1 \%$ error at all frequencies, demonstrating the controllable accuracy of the implementation. For this accuracy, the largest problem discretized with more than 50 million unknowns is solved in less than $15 \mathrm{~h}$ using approximately 400 GB total peak memory.

Figure 2 presents solutions of electromagnetics problems involving spherical composite objects with various material properties. As in the previous problems, a sphere of radius $50 \mu \mathrm{m}$ (core) is placed inside another sphere of radius $100 \mu \mathrm{m}$ (shell). The frequency is fixed to $96 \mathrm{THz}$ and three different cases are considered:

1. The core is lossy dielectric with a relative permittivity of $20+0.2 i$, whereas the shell is lossless dielectric with a relative permittivity of 10 .

2. The core is plasmonic with a relative permittivity of $-2+i$, whereas the shell is lossy dielectric with a relative permittivity of $4+0.01 i$.

3. The core has a double-negative property, i.e., its relative permittivity and relative permeability are $-10+i$ and -1 , respectively. The shell is lossless dielectric with a relative permittivity of 2 .

Each object is illuminated by a plane wave propagating in the $z$ direction with the electric field polarized in the $x$ direction. Discretizations lead to matrix equations involving $13,112,832$ unknowns. As an important advantage of using surface formulations, we do not need to refine the discretization as the contrast of the object increases. Specifically, the triangle size is fixed to $\lambda_{0} / 10$, where $\lambda_{0}$ is the wavelength in

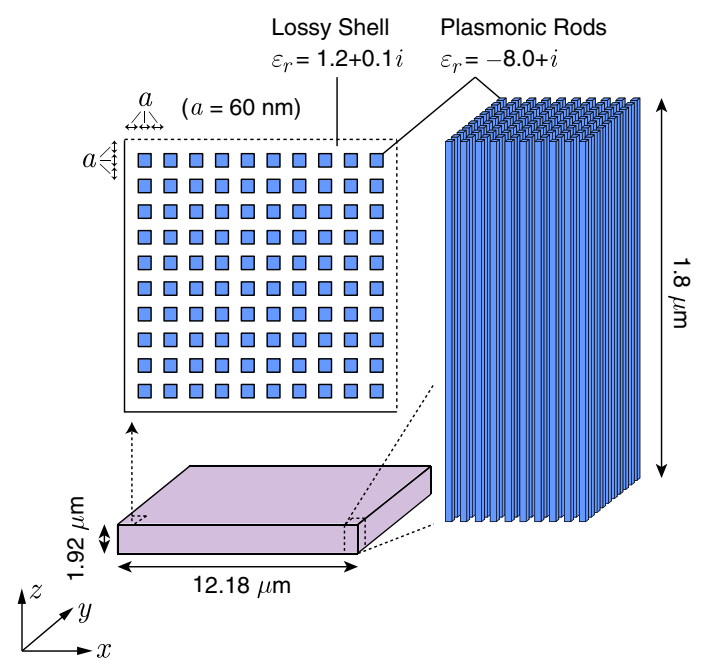

Fig. 3. (Color online) Metamaterial structure designed for optical frequencies: rectangular plasmonic rods are placed inside a dielectric box. 


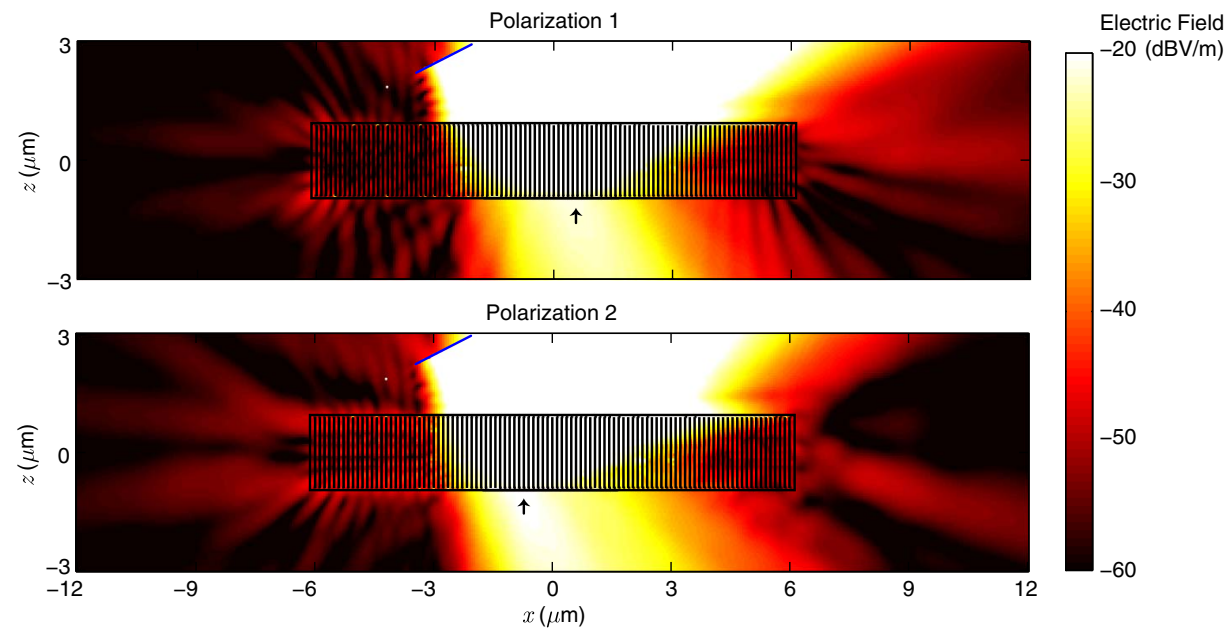

Fig. 4. (Color online) Solutions of electromagnetics problems involving an optical metamaterial structure excited with beams with two different polarizations. The total electric field in the vicinity of the structure is plotted on the $z-x$ plane. The structure exhibits positive refraction when the beam has a $\phi$-polarized magnetic field (polarization 1) and negative refraction when the beam has a $y$-polarized magnetic field (polarization 2).

the host medium that contains the excitation. The same discretization can be used for higher contrasts, provided that integrals on triangles are carried out accurately and geometric deviations due to planar modeling of smooth surfaces are negligible. Figure 2 depicts the bistatic SCS values in $\mathrm{dB} \mu \mathrm{ms}$ on the $z-x$ plane with respect to the bistatic angle from $0^{\circ}$ (forward-scattering direction) to $180^{\circ}$ (backscattering direction). Similar to previous examples, computational values obtained by using MLFMA agree very well with those obtained via analytical Mie-series solutions. Table 2 lists more quantitative information on the efficiency and accuracy of solutions. The total time is correlated with the number of iterations, which directly depends on the problem, e.g., medium parameters. In addition, it is clearly visible that the processing time and total peak memory used for a problem increase as the absolute value of the permittivity (hence the wavenumber) increases, since more samples are required for the radiated and incoming fields to compute far-field interactions. Finally, computations of near-field interactions require more time for larger wavenumber values due to more oscillatory integrands over basis and testing domains, leading to further increase in the total processing time. Despite those significant variations in the computational cost, Table 2 shows that the RMS error is consistently below $1 \%$ in all three solutions.

Figure $\underline{3}$ presents a metamaterial structure designed for negative refraction at optical frequencies, similar to those in [38,39]. A total of $101 \times 101$ rectangular rods are placed inside a dielectric box located at the origin. The size of each rod is $0.06 \mu \mathrm{m} \times 0.06 \mu \mathrm{m} \times 1.8 \mu \mathrm{m}$, while the size of the box (hence the overall structure) is $12.18 \mu \mathrm{m} \times 12.18 \mu \mathrm{m} \times$ $1.92 \mu \mathrm{m}$. The structure is investigated at $417 \mathrm{THz}$, where its discretization leads to matrix equations involving $8,216,880$ unknowns. The rods are plasmonic with a relative permittivity of $-8+i$ whereas the enclosing box is lossy with a relative permittivity of $1.2+0.1 i$. The structure is excited with electromagnetic beams created by Hertzian dipoles located at $\left(x_{d}, y_{d}, z_{d}\right)=(-1.2+1.5 i, 0,3.36-3 i) \mu \mathrm{m}$ in complex coordinates [40]. Two different polarizations are considered:
1. The dipole moment of the Hertzian dipole is in $\left(\theta_{d}, \phi_{d}\right)=\left(90^{\circ}, 90^{\circ}\right)$ direction. Hence, the created beam has a $\theta$-polarized magnetic field on the $z-x$ plane.

2. The dipole moment of the Hertzian dipole is in $\left(\theta_{d}, \phi_{d}\right)=\left(63^{\circ}, 0\right)$ direction. Hence, the created beam has a $y$-polarized magnetic field on the $z-x$ plane. For this polarization, the metamaterial is expected to demonstrate negative refraction $[\underline{38}, \underline{39}]$.

In both cases, the beam propagates in $\left(\theta^{\text {inc }}, \phi^{\text {inc }}\right) \approx\left(153^{\circ}, 0\right)$ direction. Using 400 GB peak memory, the total processing time, including the setup and two iterative solutions (a total of 190 iterations), is less than $14 \mathrm{~h}$.

Figure $\underline{4}$ depicts the total electric field in the vicinity of the metamaterial on the $z-x$ plane. The beams, which are originated at blue lines in Fig. 4 , are placed carefully such that the electric field on the input side (top) of the metamaterial slab is maximum at around $x=0$. Then, by measuring the electric field on the output side and finding the location of the maximum and its shift with respect to $x=0$ (shown with small arrows in Fig. 4), we are able to determine the properties of the refraction. Specifically, for the first polarization, the beam is shifted by approximately $0.6 \mu \mathrm{m}$ in the positive $x$ direction. For the second polarization, however, the beam is shifted by approximately $0.75 \mu \mathrm{m}$ in the negative $x$ direction, which can be interpreted as a negative refraction. These numerical results are consistent with theoretical and experimental findings [38,39], i.e., the metamaterial exhibits negative refraction depending on the polarization.

\section{CONCLUDING REMARKS}

This paper presents a parallel implementation of MLFMA for composite structures involving multiple dielectric and metallic parts. The implementation is based on

- surface formulations with JMCFIE,

- discretizations with the RWG functions,

- iterative solutions with MLFMA,

- and parallel solutions using the hierarchical partitioning strategy. 
Capabilities of the implementation are demonstrated on large-scale electromagnetics problems involving canonical and complicated geometries. We show that the implementation enables accurate and efficient solutions of composite problems that may be difficult to analyze with other methods in the literature.

\section{ACKNOWLEDGMENT}

This work was supported by the Scientific and Technical Research Council of Turkey (TUBITAK) under Research Grants 110E268 and 111E203, by the Engineering and Physical Sciences Research Council (EPSRC) under Research Grant EP/J007471/1, by the Centre for Numerical Algorithms and Intelligent Software (EPSRC-EP/G036136/1), and by contracts from ASELSAN and SSM. The authors would like to thank Mert Hidayetoğlu of the Bilkent University Computational Electromagnetics Research Center (BiLCEM) for generating the computer models of the three-dimensional optical metamaterial structures investigated in this paper.

\section{REFERENCES}

1. J. Song, C.-C. Lu, and W. C. Chew, "Multilevel fast multipole algorithm for electromagnetic scattering by large complex objects," IEEE Trans. Antennas Propag. 45, 1488-1493 (1997).

2. X.-Q. Sheng, J.-M. Jin, J. Song, W. C. Chew, and C.-C. Lu, "Solution of combined-field integral equation using multilevel fast multipole algorithm for scattering by homogeneous bodies," IEEE Trans. Antennas Propag. 46, 1718-1726 (1998).

3. W. C. Chew, J.-M. Jin, E. Michielssen, and J. Song, Fast and Efficient Algorithms in Computational Electromagnetics (Artech House, 2001).

4. S. Velamparambil, W. C. Chew, and J. Song, "10 million unknowns: Is it that big?, IEEE Antennas Propag. Mag. 45(2), 43-58 (2003).

5. S. Velamparambil and W. C. Chew, "Analysis and performance of a distributed memory multilevel fast multipole algorithm," IEEE Trans. Antennas Propag. 53, 2719-2727 (2005).

6. L. Gürel and Ö. Ergül, "Fast and accurate solutions of extremely large integral-equation problems discretised with tens of millions of unknowns," Electron. Lett. 43, 499-500 (2007).

7. Ö. Ergül and L. Gürel, "Hierarchical parallelisation strategy for multilevel fast multipole algorithm in computational electromagnetics," Electron. Lett. 44, 3-5 (2008).

8. X.-M. Pan and X.-Q. Sheng, "A sophisticated parallel MLFMA for scattering by extremely large targets," IEEE Antennas Propag. Mag. 50(3), 129-138 (2008).

9. Ö. Ergül and L. Gürel, "Efficient parallelization of the multilevel fast multipole algorithm for the solution of large-scale scattering problems," IEEE Trans. Antennas Propag. 56, 2335-2345 (2008).

10. J. Fostier and F. Olyslager, "An asynchronous parallel MLFMA for scattering at multiple dielectric objects," IEEE Trans. Antennas Propag. 56, 2346-2355 (2008).

11. J. Fostier and F. Olyslager, "Provably scalable parallel multilevel fast multipole algorithm," Electron. Lett. 44, 11111113 (2008).

12. J. Fostier and F. Olyslager, "Full-wave electromagnetic scattering at extremely large 2-D objects," Electron. Lett. 45, 245-246 (2009).

13. Ö. Ergül and L. Gürel, "A hierarchical partitioning strategy for an efficient parallelization of the multilevel fast multipole algorithm," IEEE Trans. Antennas Propag. 57, 1740-1750 (2009).

14. M. G. Araujo, J. M. Taboada, F. Obelleiro, J. M. Bertolo, L. Landesa, J. Rivero, and J. L. Rodriguez, "Supercomputer aware approach for the solution of challenging electromagnetic problems," Prog. Electromagn. Res. 101, 241-256 (2010).

15. J. M. Taboada, M. G. Araujo, J. M. Bertolo, L. Landesa, F. Obelleiro, and J. L. Rodriguez, "MLFMA-FFT parallel algorithm for the solution of large-scale problems in electromagnetics," Prog. Electromagn. Res. 105, 15-30 (2010).

16. J. Fostier and F. Olyslager, "An open-source implementation for full-wave 2D scattering by million-wavelength-size objects," IEEE Antennas Propag. Mag. 52(5), 23-34 (2010).

17. X.-M. Pan, W.-C. Pi, and X.-Q. Sheng, "On OpenMP parallelization of the multilevel fast multipole algorithm," Prog. Electromagn. Res. 112, 199-213 (2011).

18. Ö. Ergül and L. Gürel, "Rigorous solutions of electromagnetics problems involving hundreds of millions of unknowns," IEEE Antennas Propag. Mag. 53(1), 18-27 (2011).

19. V. Melapudi, B. Shanker, S. Seal, and S. Aluru, "A scalable parallel wideband MLFMA for efficient electromagnetic simulations on large scale clusters," IEEE Trans. Antennas Propag. 59, 2565-2577 (2011).

20. J. Fostier, B. Michiels, I. Bogaert, and D. De Zutter, "A fast 2-D parallel multilevel fast multipole algorithm solver for oblique plane wave incidence," Radio Sci. 46, RS6006 (2011).

21. Ö. Ergül, "Solutions of large-scale electromagnetics problems involving dielectric objects with the parallel multilevel fast multipole algorithm," J. Opt. Soc. Am. A 28, 2261-2268 (2011).

22. Ö. Ergül, "Parallel implementation of MLFMA for homogeneous objects with various material properties," Prog. Electromagn Res. 121, 505-520 (2011).

23. Ö. Ergül and L. Gürel, "Accurate solutions of extremely large integral-equation problems in computational electromagnetics," Proc. IEEE 101, 342-349 (2013)

24. P. Ylä-Oijala and M. Taskinen, "Application of combined field integral equation for electromagnetic scattering by dielectric and composite objects," IEEE Trans. Antennas Propag. 53, 1168-1173 (2005).

25. P. Ylä-Oijala, M. Taskinen, and S. Järvenpää, "Surface integral equation formulations for solving electromagnetic scattering problems with iterative methods," Radio Sci. 40 RS6002 (2005).

26. Ö. Ergül and L. Gürel, "Comparison of integral-equation formulations for the fast and accurate solution of scattering problems involving dielectric objects with the multilevel fast multipole algorithm," IEEE Trans. Antennas Propag. 57, 176-187 (2009).

27. Ö. Ergül and L. Gürel, "Efficient solution of the electric and magnetic current combined-field integral equation with the multilevel fast multipole algorithm and block-diagonal preconditioning," Radio Sci. 44, RS6001 (2009).

28. J. Rivero, J. M. Taboada, L. Landesa, F. Obelleiro, and I. GarciaTunon, "Surface integral equation formulation for the analysis of left-handed metamaterials," Opt. Express 18, 15876-15886 (2010).

29. Ö. Ergül, "Fast and accurate analysis of homogenized metamaterials with the surface integral equations and the multilevel fast multipole algorithm," IEEE Antennas Wirel. Propag. Lett. 10, 1286-1289 (2011).

30. Ö. Ergül, "Analysis of composite nanoparticles with surface integral equations and the multilevel fast multipole algorithm," J. Opt. 14, 062701 (2012).

31. S. M. Rao, D. R. Wilton, and A. W. Glisson, "Electromagnetic scattering by surfaces of arbitrary shape," IEEE Trans. Antennas Propag. 30, 409-418 (1982).

32. P. Ylä-Oijala, M. Taskinen, and J. Sarvas, "Surface integral equation method for general integral equation method for general composite metallic and dielectric structures with junctions," Prog. Electromagn. Res. 52, 81-108 (2005).

33. S. Ohnuki and W. C. Chew, "Truncation error analysis of multipole expansion," SIAM J. Sci. Comput. 25, 1293-1306 (2004).

34. R. Coifman, V. Rokhlin, and S. Wandzura, "The fast multipole method for the wave equation: a pedestrian prescription," IEEE Antennas Propag. Mag. 35(3), 7-12 (1993).

35. Ö. Ergül, I. van den Bosch, and L. Gürel, "Two-step Lagrange interpolation method for the multilevel fast multipole algorithm," IEEE Antennas Wirel. Propag. Lett. 8, 69-71 (2009).

36. A. Brandt, "Multilevel computations of integral transforms and particle interactions with oscillatory kernels," Comput. Phys. Commun. 65, 24-38 (1991). 
37. H. van der Vorst, "Bi-CGSTAB: A fast and smoothly converging variant of Bi-CG for the solution of nonsymmetric linear systems," SIAM J. Sci. Stat. Comput. 13, 631-644 (1992).

38. J. Yao, Z. Liu, Y. Liu, Y. Wang, C. Sun, G. Bartal, A. M. Stacy, and $\mathrm{X}$. Zhang, "Optical negative refraction in bulk metamaterials of nanowires," Science 321, 930 (2008).
39. Y. Liu, G. Bartal, and X. Zhang, "All-angle negative refraction and imaging in a bulk medium made of metallic nanowires in the visible region,” Opt. Express 16, 15439-15448 (2008).

40. K. Tap, "Complex source point beam expansions for some electromagnetic radiation and scattering problems," $\mathrm{Ph} . \mathrm{D}$. dissertation (The Ohio State University, 2007). 